\title{
RELIABILITY OF LED'S; ARE THE ACCELERATED AGEING TESTS RELIABLE?
}

\author{
GEORGE FERENCZI \\ Research Institute for Technical Physics of the HAS 1325 Budapest, Ujpest 1, P.O. Box 76 Hungary \\ (Received April 16, 1981; in final form October 20, 1981)
}

\begin{abstract}
The mechanisms, leading to light output degradation of LED's were studied using a number of different techniques e.g. deep level spectroscopy, electroluminescence, minority carrier life time measurements. Several processes were revealed having different temperature and stress current dependence. Using these data extrapolations based on accelerated ageing test results are reexamined.
\end{abstract}

\section{INTRODUCTION}

This paper covers gradual performance degradation caused by processes in the bulk of the semiconductor devices. The surface effects meaning contacts, oxide coverings, catastrophal failure etc. are outside the scope of the present paper.

The results that are presented are based on investigations on $\mathrm{GaP}$ based light emitting diodes. The conclusions are easily generalized for other compound semiconductor devices but their relevance for $\mathrm{Si}$ based devices is not straightforward. It is hoped, however, that some of the conclusions are relevant for high current density $\mathrm{Si}$ devices such as used in VLSI circuitry.

The structure of this paper is as follows: An interpretive background of the possible physical processes is given followed by some specific experiments on LED's. A critical evaluation of the scale laws used for accelerated ageing tests closes the paper.

\section{BACKGROUND}

The performance degradation is mainly concerned with the formation of new non-radiative recombination sites leading to a decrease of the radiative quantum efficiency. This process is obviously prohibited in light emitting devices i.e. all effort should be concentrated to avoid it. The formation of nonradiative recombination centres increases the high frequency noise with serious consequences for microwave semiconductor devices.

If these centres form at interfaces the increased interface density of states could lead to erratic switching properties, a drawback for usual integrated circuitry. Finally the formation of non-radiative centres is a symptom of dislocation movement and increased dislocation concentration. In extreme cases the increased dislocation concentration builds up mechanical stress fields resulting in catastrophic failure.

One of the first degradation models was developed for tunnel diodes in the early sixties by Longini. ${ }^{1} \mathrm{He}$ assumed that the electrical field and current transport in the diodes may cause ion migration resulting in modified impurity distribution. This model was considered as a possible explanation for a long period without establishing direct evidence. Another model put forward by Gold and Weissberg ${ }^{2}$ claimed that the energy liberated in non-radiative recombination could cause impurity migration by a phonon-kick mechanism.

The main observation that both models is based on is valid. Degradation is a consequence of forward biasing. Bulk degradation was never observed in reserve biased or non-biased devices kept under different environmental conditions.

No unified degradation model existed until now; working hypotheses were established and some of them could explain the majority of the observations. Before describing the models it is necessary to emphasize that these models were created for compound semiconductor where the presence of two different species in the host lattice and the relative nonstochiometry is of fundamental importance, hence the individual mechanisms are not relevant in silicon. The very recent results by Watkins ${ }^{3}$ on the properties of single vacancies in $\mathrm{Si}$ indicate that certain charge states of the $\mathrm{V}_{\mathrm{Si}}$ are not stable leading to the so called Anderson negative $U$ process. ${ }^{4}$ This is an athermal process with similar consequences as the findings for compound semiconductors.

The most popular model for compound semiconductors is based on the multi phonon emission model. ${ }^{5}$ This assumes that during non-radiative recombination the recombination site is adiabatically 
decoupled from the rest of the lattice and the energy liberated during recombination has no chance to dissipate uniformly via phonon coupling in the whole lattice. Instead large lattice relaxation occurs. As a consequence of this lattice relaxation defect reactions such as defect dissociation, creation or migration could happen. Each of these reactions can be thermally activated but the usual thermal activation energy is high - in the order of electron Volts - i.e. their probability is extremely low at normal operational temperatures. However the energy liberated during recombination lowers the thermal barrier to such an extent that the above described defect reactions become viable at operational temperatures. This is why these mechanisms are called Recombination Enhanced Defect Reactions. The energy liberated in a recombination process depends on the depths of the recombination sites. The energy liberated usually increases with increased band gap. In $\mathrm{GaP}$ with a band gap of $2.2 \mathrm{eV}$ the recombination energy might be larger than the thermal reaction barrier and hence athermal REDR mechanism is expected and was experimentally observed. ${ }^{\circ}$

The basic assumption for REDR processes is that the reaction sites are recombination centers. This assumes - let us confine ourselves to the example of an electron trap in an n-type material - that the hole capture cross section $\sigma_{\mathrm{p}}$ is larger than the electron capture cross section $\sigma_{\mathrm{n}}$, since the recombination rate is proportional to $\sigma_{\mathrm{p}} \mathrm{p} / \sigma_{\mathrm{n}} \mathrm{n}$. If $\sigma_{\mathrm{p}}>\sigma_{\mathrm{n}}$ is not valid then the rate of a REDR process could be influenced by the variation of the number of holes i.e. by the forward current density.

\section{EXPERIMENTAL RESULTS}

The experiments were carried out on commercial VPE GaP: $\mathrm{N}^{+}-\mathrm{n}$ light emitting diodes. The diodes were mounted on a T0-18 header without encapsulation. The ageing experiments were carried out at room temperature at different stress temperatures. The light output, the minority carrier life time and the deep level spectra of the samples were regularly monitored using a high sensitivity lock-in type spectrometer. $^{7}$

The ageing experiments at room temperature revealed, that a single deep level $-\mathrm{T}_{6}-$ is responsible for the observed light output reduction. ${ }^{8}$ As is demonstrated on Figure $1 \mathrm{~T}_{6}$ is not present in the virgin diodes yet it appears with current stressing. It was found that $\mathrm{T}_{6}$ is a recombination centre with $\sigma_{\mathrm{n}}=2 \times 10^{-16} \mathrm{~cm}^{2}, \sigma_{\mathrm{p}}=10^{-14} \mathrm{~cm}^{2}$ and the uncor-

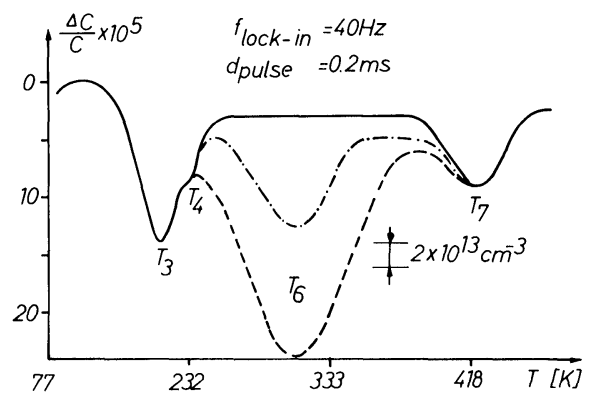

FIGURE 1 Reduced reverse bias spectrum. The diode was stressed at $25^{\circ} \mathrm{C}$ and $160 \mathrm{~A} / \mathrm{cm}^{2} \mathrm{dc}$. Ageing times: - Oh, . $-83 \mathrm{~h},---830 \mathrm{~h}$

rected thermal activation energy is $0.49 \mathrm{eV}$ from the conduction band. Using the minority carrier lifetimes efficiency variation data Figure 2 we assumed a single centre model i.e. the radiative minority carrier life time remains unaltered during ageing and the opening-up of a single non-radiative channel is completely responsible for the observed efficiency reduction. The experimental results verified these assumptions (Figure 3). The picture becomes more complicated however when the current stressing is done at elevated temperatures. Figure 4 shows the influence of the ambient temperature on the DLTS spectra. All experiments were done at $32 \mathrm{~A} / \mathrm{cm}^{2}$ current level to avoid overheating of the junctions. The " $\mathrm{T}_{6}$ " peak position shifts toward higher temperatures as the ambient temperature increases during ageing. The concentration of $T_{6}$ is about the same in each case and does not correlate any longer with the decrease of the quantum efficiency. This is also clearly demonstrated on the minority carrier versus quantum efficiency plot (Figure 5). The minority carrier life time data and the DLTS results bring information from the n-side only hence we have to

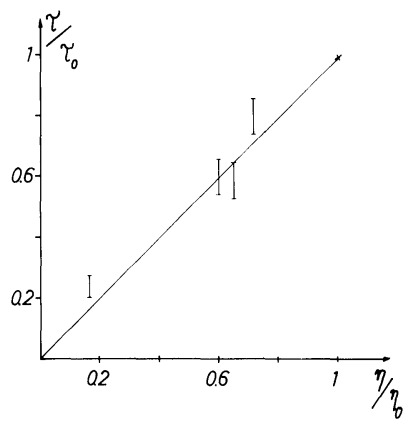

FIGURE 2 Normalized minority carrier lifetime versus normalized efficiency. The measurements were carried out on a diode aged at room temperature at $320 \mathrm{~A} / \mathrm{cm}^{2}$. Subsequent thermal anneal removed the efficiency saturation. 


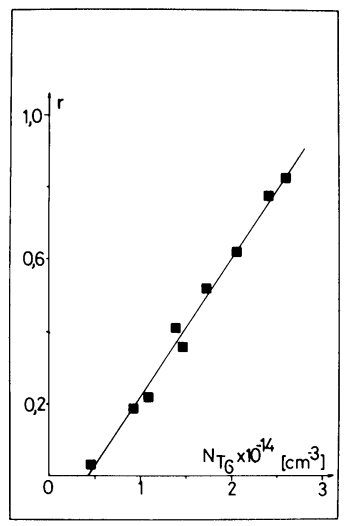

FIGURE 3 Experimental verification of the single dominant non-radiative channel model expressed by the equation: $\mathrm{r}=\mathrm{f}_{\mathrm{n} / \mathrm{n}_{0}}-1=\tau_{0} \sigma_{\mathrm{p}} \mathrm{vN}_{\mathrm{T}_{6}}$ where $\mathrm{f}_{\mathrm{n} / \mathrm{n}_{\mathrm{o}}}$ is the normalized efficiency, $\tau_{0}$ is the minority carrier life-time of the virgin diode, $\sigma_{\mathfrak{p}}$ is the hole capture cross section, $v$ is the thermal drift velocity and $\mathrm{N}_{\mathrm{T}_{6}}$ denotes the measured trap concentration.

assume that an alternative degradation process should take place at the p-side of the junction. This process is inactive or at least much slower at room temperature than the $\mathrm{T}_{6}$ trap formation mechanism.

\section{SCALE LAWS}

The results described in the previous section give a method for establishing scale laws. Scale laws are the most important prerequisite of any accelerated ageing test, since real time life tests under operational circumstances are practically impossible keeping in mind that the average device life times are often above 5 years. Accelerated ageing tests are carried out

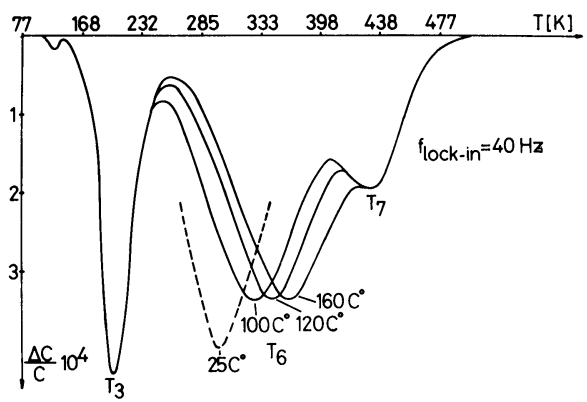

FIGURE 4 The influence of the ambient temperature during current stressing on the DLTS spectra. The samples where stressed at $32 \mathrm{~A} / \mathrm{cm}^{2} \mathrm{dc}$. for 5 hours. The light output reduced to $83 \%$ at $100^{\circ} \mathrm{C}, 79 \%$ of $120^{\circ} \mathrm{C}$ and $54 \%$ at $160^{\circ} \mathrm{C}$. The dashed curve schematically illustrates the $\mathrm{T}_{6}$ peak position at room temperature ageing experiments.

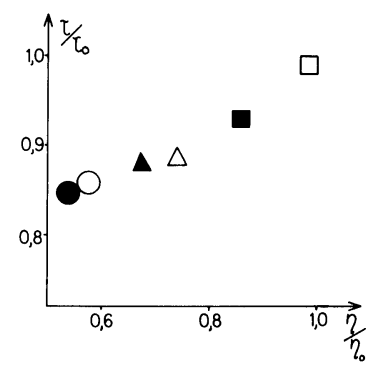

FIGURE 5 Normalized minority carrier life-time versus normalized efficiency data on diodes stressed for 5 hours at $32 \mathrm{~A} / \mathrm{cm}^{2} \mathrm{dc}$ current however at different ambient temperatures. $\square-25^{\circ} \mathrm{C},-100^{\circ} \mathrm{C}, \Delta-120^{\circ} \mathrm{C}, \triangle-140^{\circ} \mathrm{C}, \circ-160^{\circ} \mathrm{C}$, - $-180^{\circ} \mathrm{C}$. The size of the symbols is to be regarded as error bars indicating that the data are average over 10 diodes at each temperature.

at elevated temperatures or higher than operational current levels or both.

If a degradation process is thermally activated more than one mechanism with different activation energies might be active simultaneously. If a process is dominant at $160^{\circ} \mathrm{C}$ it is not necessarily dominant at room temperature. The previous experimental results on green GaP LED's provide a good example for this. The $\mathrm{T}_{6}$ trap formation mechanism which is explained in detail in reference 9 where the details of our alternative model for large lattice relaxation will be presented. Only the relevant results are quoted here. $\mathrm{T}_{6}$ forms as a vacancy clusters from vacancies emitted by electron-hole recombination propelled dislocation climb. The large energy liberated during a non-radiative recombination process (in the order of $2 \mathrm{eV}$ ) is sufficient for the cluster formation which structure is formed to minimize local shear stresses in the lattice. This structure is however thermodynamically in non-equilibrium i.e. this is why the thermal energy at elevated temperature ageing tends to destroy the metastable structure created by the recombination energy. As a result the defect formation rate and hence the degradation rate might be quite different at elevated temperature than at room temperature.

An even stronger evidence was found by Shimano. ${ }^{10}$ His results show a negative activation energy above $180^{\circ} \mathrm{C}$ and a positive below this temperature.

If $\sigma_{\mathrm{p}}>\sigma_{\mathrm{n}}$ does not hold for a non-radiative centre additional complication arises, the REDR rate becomes injection level dependent. Increasing the current level, the $p-n$ junction temperature is increased as well. The REDR rate as well as the degradation rate will increase as a combination of two separate influences.

These examples provided evidence against any straightforward extrapolation from accelerated ageing 
test results. It has to be emphasized again that the results presented here were on III-V semiconductor devices. The recombination energy liberated in these materials is much larger than in silicon devices and the equilibrium concentration of point defects is much larger owing to the inherent non-stochiometry of the compound semiconductors. The situation is much less serious for silicon devices as far as the validity of extrapolation from accelerated test results is concerned.

\section{CONCLUSIONS}

The analysis of ageing test results on commercial GaP LED's leads us to thermodynamical considerations which oppose any straightforward extrapolations from elevated temperature ageing results. The main argument is that the large energy $(\sim 2 \mathrm{eV})$ liberated in non-radiative recombination processes might create defect configurations which are metastable i.e. nonequilibrium structures and so defect migration at elevated temperatures tends to destroy them.
Extrapolations are only justified if the actual degradation mechanism is known. Deep level spectroscopy combined with minority carrier life-time measurement is an excellent tool to provide the required insight into the defect reaction. The individual REDR rates can be determined for each defect and so the true extrapolation for operational circumstances can be established.

\section{REFERENCES}

1. R.L. Longini: Solid St. Electron, 5, 127 (1962).

2. B.D. Gold and L.R. Weissberg: Solid St. Electron, 7, 811 (1964).

3. G.B. Watkins and J.R. Troxell: Phys. Rev. Letters 44, 593 (1980).

4. P.W. Anderson: Phys. Rev. Lett. 34, 953 (1975).

5. C.H. Henry and D.V. Lang: Phys. Rev. B. 15, 989 (1977).

6. D.V. Lang and L.C. Kimerling: Appl. Phys. Letters, 28, 248 (1976).

7. G. Ferenczi and J. Kiss, Acta Phys. Hung. 50, 285 (1981).

8. G. Ferenczi: IEEE Trans. on Electron. Dev. ED-28, 421 (1981).

9. G. Ferenczi, F. Beleznay and L. Dózsa: to be published.

10. N. Shimano, J. Appl. Phys. 51, 1818 (1980). 

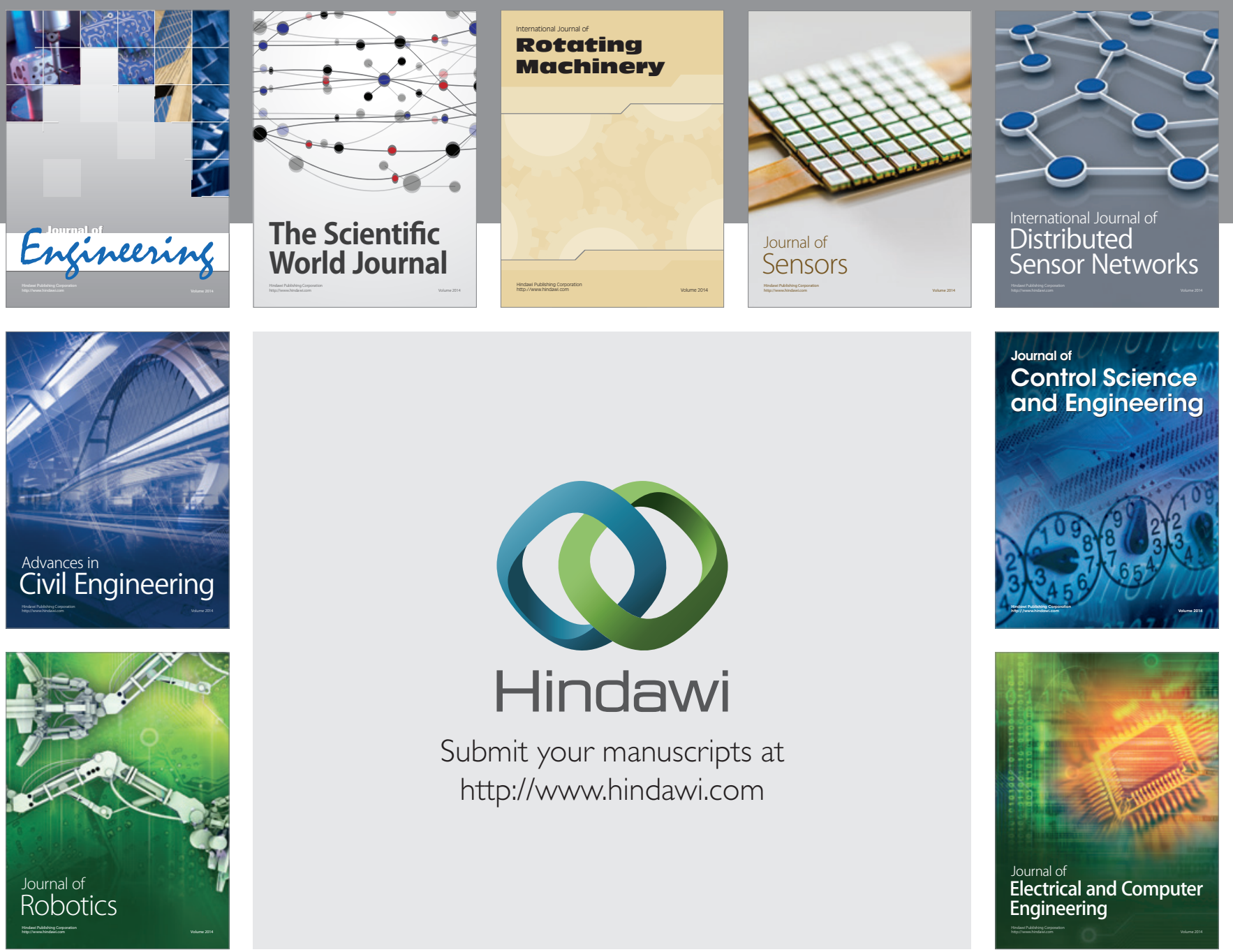

Submit your manuscripts at

http://www.hindawi.com
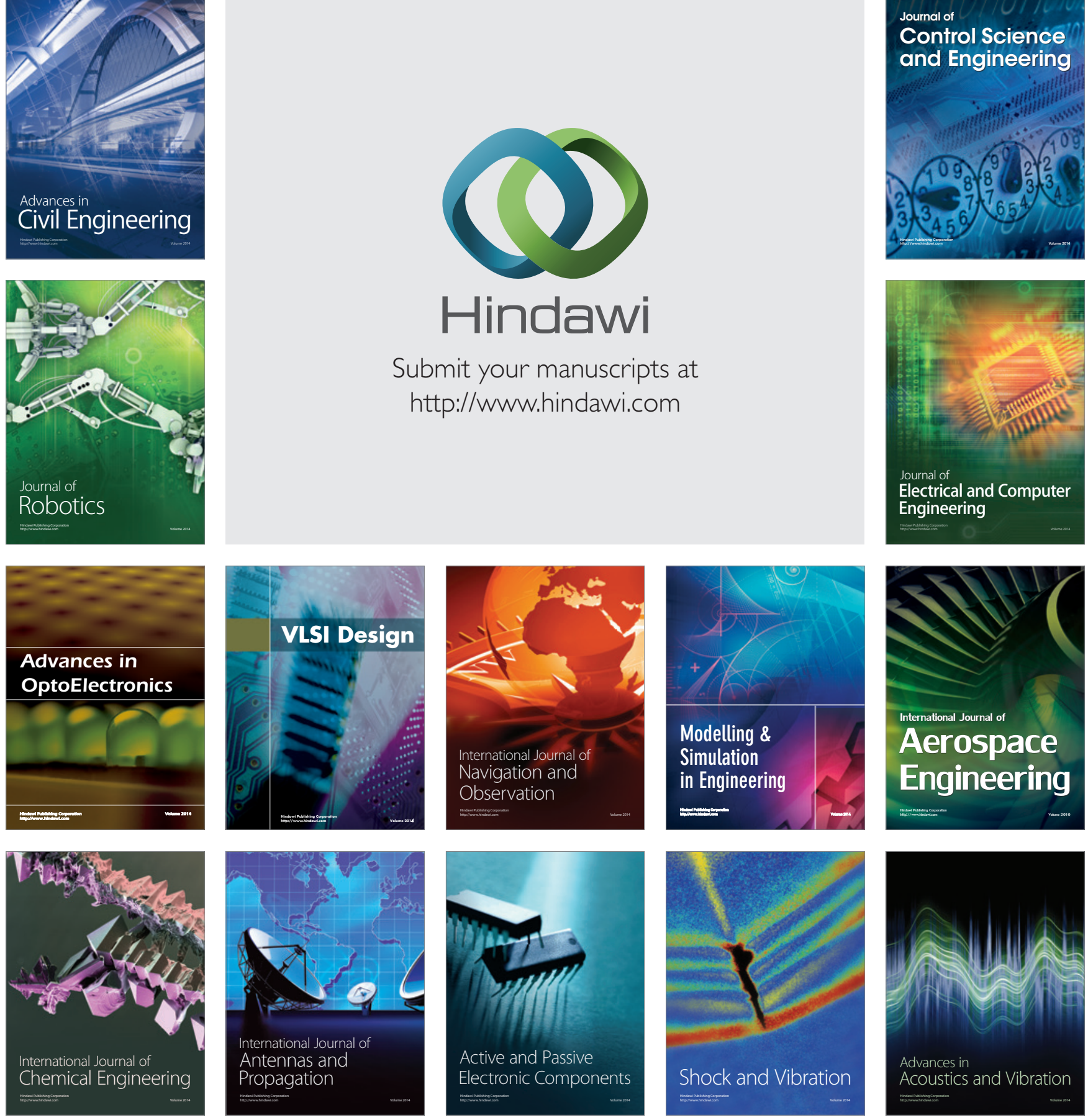